\title{
Project, Construction and Test of a Mini Computerized Tomograph
}

\author{
J. M. de Oliveira Jr. \\ Centro de Ciências Exatas e Tecnlógicas - CETEC, \\ Universidade de Sorocaba-UNISO, Cidade Universitária, \\ 18023-000, C.P. 578, Sorocaba, SP, Brasil
}

Received on 30 October, 2002

\begin{abstract}
Computed Tomography (CT) refers to the cross-sectional imaging of an object from both transmission or reflection data collected by illuminating the object from many different directions. CT is an imaging technique that has revolutionized the field of medical diagnostics. There are many others applications for CT images, such as, nondestructive evaluation of industrial products and analysis of biological specimens. A mini computerized tomograph was projected, constructed and tested. It operates with a gamma ray source of ${ }^{241} \mathrm{Am}$ (photons of $60 \mathrm{keV}$ and $100 \mathrm{mCi}$ of intensity) and a $\mathrm{NaI}(\mathrm{Tl})$ solid state detector. The system features translation and rotation scanning modes, a $100 \mathrm{~mm}$ effective field of view, and $1 \mathrm{~mm}$ spatial resolution. The preliminary results indicated a resolution between $5 \%$ to $7 \%$ to detect mass attenuation coefficient variations. The total cost of the Mini Computerized Tomograph of UNISO (MTCU) was about US\$20.000,00.
\end{abstract}

\section{Introduction}

The fundamental principle behind CT, or image reconstruction from projections, has been know since the studies made by Radon [1] in 1917, in which the Radon inversion formula was derived and proved. Some of the important publications are the works of Cormack [2,3] on the representations of a function by its line integrals; Bracewell and Riddle [4] on the reconstruction of brightness distribution of astronomical bodies from fan-beam scans at various angles; De Rosier and Klug $[5,6]$ on the reconstruction of three dimensional images of virus. Ramachandran and Lakshminarayanan [7] on the convolution backprojection technique. The algebraic approach was described by Gordon et al. [8]. The early works on the development of practical scanners for medical applications were performed by Oldendorf [9], Hounsfield [10], and Ambrose [11]. The mathematical problem of the CT imaging is that of estimating an image from its projections measured at different angles and directions [12, 13]. Computerized tomography has found its most important application in medicine, but has also been applied to nondestructive testing of materials and industrial objects. This article describes how the mini computerized tomograph constructed at UNISO operates, see Fig. 1 and presents some discussion on the technique employed in the reconstruction images, used in the first test of UNISO's tomograph.

\section{Projection data and experimental setup}

The mathematical algorithms for tomographic reconstruction are based on projection data. These projections can

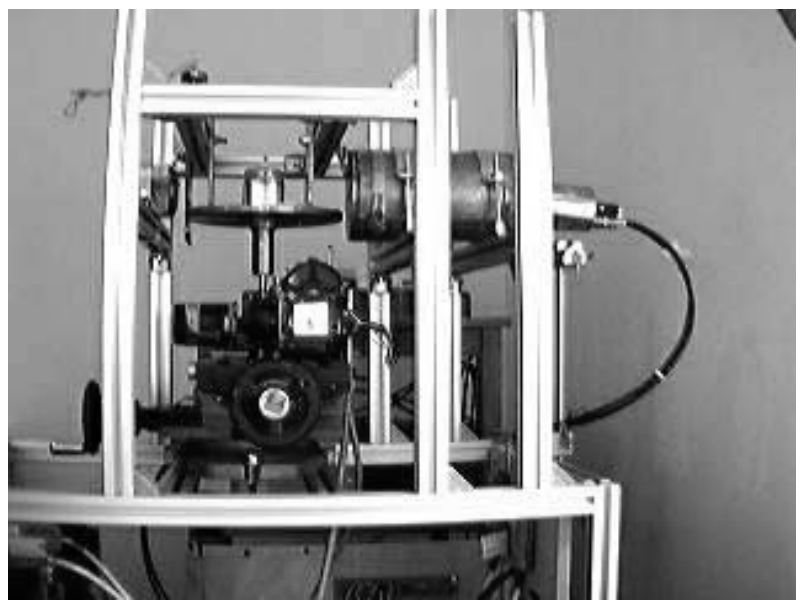

Figure 1. Elements of an $\gamma$-ray transmission computed tomography system of UNISO.

represent, for example, the linear attenuation coefficient of $\gamma$-rays along the path of the ray. The attenuation of an $\gamma$ rays beam is dependent on the energy of the photon, as well as the density of the object or its constituents along the ray path. Let $\mathrm{N}_{i}$ denotes the number of $\gamma$-ray photons incident upon the body within a specified time interval for a particular ray path. Let $\mathrm{N}$ be the corresponding number of photons exiting the body. Then, we can write for a mono-energetic $\gamma$-ray beam

$$
\int \mu(x, y) d s=\ln \left(\frac{N_{i}}{N}\right)
$$

where $\mathrm{N}_{i}$ and $\mathrm{N}$ are Poisson variables, $\mu(\mathrm{x}, \mathrm{y})$ represents the linear attenuation coefficient at $(\mathrm{x}, \mathrm{y})$, ds represents the elemental distance along the ray, and the integral is along the 
ray path from the $\gamma$-ray source to the detector. A measurement of the incident and the exiting $\gamma$-ray thus gives only an integral of $\mu(\mathrm{x}, \mathrm{y})$ over the ray path. The details of the body along the ray path are summed on to a single measurement. Extending the same argument to all ray paths, we can use the principle of image reconstruction from projections, to compute an image of a section of the body. The MTCU uses the same principle as the first generation medical tomograph (i. e. translate-rotate scanning geometry for parallel-ray projection), but with one difference, the source and the detector are fixed and the body to be analyzed moves. The image of a section of the object is obtained constructing an imaginary image matrix of this section, composed of elements of predetermined size. The matrix image must be sufficiently large to encompass the body to be imaged. In the case of a body with $50 \mathrm{~mm}$ of radio, typically 100 elements are used to span these $50 \mathrm{~mm}$. To obtain the transmission data from which the image is formed, an aligned $\gamma$-ray source-detector arrangement is used. This system is highly collimated to the width of one image element. The initial transverse part of the scanning is a linear motion in which each of the 100 or more separate data points are entered into the computer during acquisition. After each single traverse, the object is rotated a preset angular increment ( $1^{0}$ for example) and the linear, transverse scan motion starts again. If 180 angular projections are used, the result is 180 times 100 or 18.000 separate projections having been entered into the computer. Data in digital form are stored in the computer as the scan is in progress. The information density of the final image is statistical controllable, by modifying the radiation quantity that must be acquired. Typically, the number of $\gamma$-ray photons used in MTCU during a projections acquisition is between 2.000 to 10.000 counts. Once the projections are obtained, we can use image reconstruction techniques to get a cross-sectional image of the object.

\section{Algebraic reconstruction}

The reconstruction problem can be solved using several methods. To reconstruct the images of the tomograph, it was used Algebraic Reconstruction Techniques(ART) [8, 14, 15, $16,17,18,19,20,21,22]$. ART is related to the Kaczmarz [23, 24] method of projections for solving simultaneous equation. The Kacsmarz method takes an approach where the available projections (ray sums in the discrete case) are seen as a set of simultaneous equations, with the unknown quantities being discrete pixels of the image. The large sizes of images encountered in practice precludes the use of the usual methods for solving simultaneous equations. The Kaczmarz method of projection is an iterative method, which may easily be implemented [12]. Let the image to be reconstructed be divided into $\mathrm{N}$ cells, with $\mathrm{f}_{j}$ denoting the value of density or attenuation mass coefficient in the jth cell (in each cell the function $\mathrm{f}_{j}$ is assumed to be constant). Let $\mathrm{p}_{i}$ be the ray-sum measured with the ith ray. The relationship between the ray-sums and densities may be expressed as

$$
p_{i}=\sum_{j=1}^{N} w_{i j} f_{j}, \quad i=1,2, \ldots, M
$$

where $\mathrm{M}$ is the total number of rays (in all the projections) and $\mathrm{w}_{i j}$ is the weighting factor that represents the contribution of the $\mathrm{jth}$ cell to the ith ray sum. The factor $\mathrm{w}_{i j}$ is equal to the fractional area of the jth image cell intercepted by the ith ray. If $\mathrm{M}$ and $\mathrm{N}$ were small, we could use conventional matrix theory methods to invert the system of equations in (2). However, in practice $\mathrm{N}$ and $\mathrm{M}$ are large which precludes any possibility of direct matrix inversion. A grid representation with $\mathrm{N}$ cells gives the image $\mathrm{N}$ degrees of freedom. Thus an image represented by $\left(\mathrm{f}_{1}, \mathrm{f}_{2}, \mathrm{f}_{3}, \ldots ., \mathrm{f}_{N}\right)$ may be considered to be a single point in an $\mathrm{N}$-dimensional hyperspace. In this space each of the above equations represents a hyperplane. When a unique solution to these equations exists, the intersection of all these hyperplanes is a single point giving that solution. To arrive at the solution, the Kaczmarz method takes the approach of successively and iteratively projecting an initial guess and its successors from one hyperplane to the next. We could write the solution at the pixel level as

$$
f_{m}^{(j)}=f_{m}^{(j-1)}+\left[\frac{\left(p_{j}-q_{j}\right)}{\sum_{k=1}^{N} w_{j k}^{2}}\right] w_{j m},
$$

where $q_{j}=\sum_{k=1}^{N} f_{k}^{(j-1)} w_{j k}$. This equation says that when we project the $(j-1)$ th estimate on to the jth hyperplane, the correction factor for the mth cell is

$$
\Delta f_{m}^{(j)}=f_{m}^{(j)}-f_{m}^{(j-1)}=\left[\frac{\left(p_{j}-q_{j}\right)}{\sum_{k=1}^{N} w_{j k}^{2}}\right] w_{j m}
$$

Here, $\mathrm{p}_{j}$ is the given (true) ray sum for the jth ray, and $\mathrm{q}_{j}$ is the computed ray sum for the same ray for estimated image on hand.The difference $\left(\mathrm{p}_{j}-\mathrm{q}_{j}\right)$ is the error in the estimate, which may be normalized and applied as a correction to all the pixel with appropriate weighting.

\section{Results and discussion}

Fig. (2) shows the first tomographic image obtained by using the mini computerized tomograph of UNISO. This image, represents a cross-section of a $3 \mathrm{D}$ aluminium object of $5 \mathrm{~cm} \times 5 \mathrm{~cm}$ showed in Fig. (3). The reconstructed image was obtained using ART (3 interactions and additive method) implementation. This reconstruction was carried out on a $50 \times 50$ sampling lattice with 100 projections per angle, from $0^{0}$ to $180^{\circ}$ in steps of $10^{\circ}$. In equation (2), this corresponds to 2500 picture elements and an overall number of rays equal 1800 . Note that the system of equations is underdetermined, but the reconstruction still of good quality. When the number of ray sums is greater than the number of pixels, i.e. $\mathrm{M}>\mathrm{N}$ in eq. (2) and the projections corrupted by noise, no unique solution exists. If $\mathrm{M}<\mathrm{N}$, the system is 
under-determined and an infinite number of solutions will exist. The major advantage of ART is that any a priori information about the image may be introduced easily into the iterative procedure. This may help in obtaining a useful solution even if the system is under-determined. The image showed in Fig. (2) was displayed using a new computer program named Tomografia [25], especially developed to be use in the UNISO's tomograph.

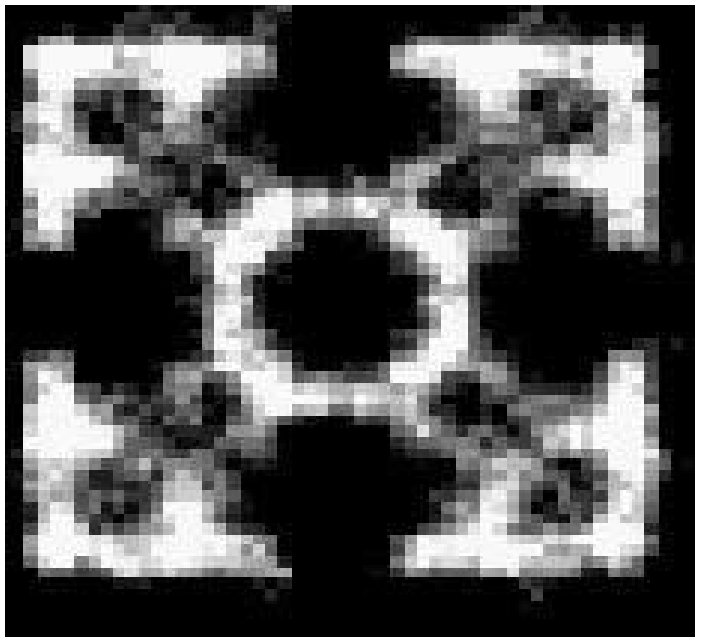

Figure 2. Reconstruction of the aluminium cross-section, obtained using 100 projections from $0^{\circ}$ to $180^{\circ}$ in steps of $10^{\circ}$ with the ART algorithm.

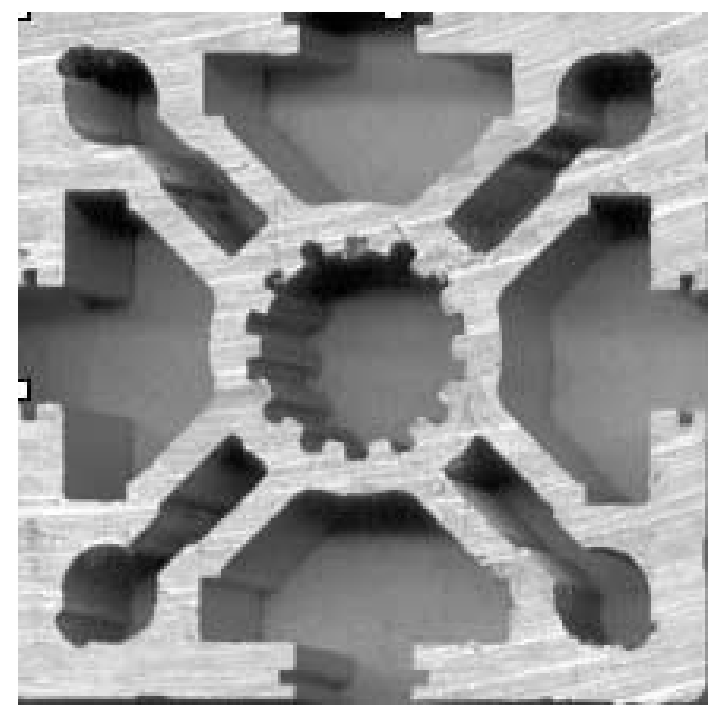

Figure 3. The cross-section of 3D real object (aluminium of $5 \mathrm{~cm}$ $\times 5 \mathrm{~cm}$ ) used in tomographic.

\section{Conclusions}

It was developed a system that can be used to obtain non destructive images of little objects. This system was denominated Mini Computerized Tomograph of UNISO (MTCU). The MTCU was tested and the first results using ART technique to reconstruct the image were very promising. Other reconstruction techniques, such as Discrete Filtered Backprojection and Fourier Transform are being implemented. New tests need to be made in order to find the best parameters to operate the tomograph.

\section{Acknowledgments}

We are grateful to FAPESP for financial support and we thank A. C. G. Martins for the many discussions.

\section{References}

[1] J. Radon, Math. Phys. K. 69, 262 (1917).

[2] A. M. Cormack, J. Appl. Phys. 34, 2722 (1963).

[3] A. M. Cormack J. Appl. Phys. 35, 2908 (1964).

[4] R. N. Bracewell and A. C. Riddle Astron. J. 150, 297 (1967)

[5] R. A. Crowther, L. A. Amos, J. T. Finch, D. J. De Rosier and A. Klug Nature 226, 421 (1970)

[6] D. J. De Rosier and A. Klug Nature 217, 130 (1968)

[7] G. N. Ramachandran and A. V. Lakshminarayanan Proc. Natl. Acad. Sci. USA 68, 2236 (1971)

[8] R. Gordon, R. Bender and G. T. Herman J. Theor. Biol. 29, 471 (1970)

[9] W. H. Oldendorf I.R.E. Trans. Bio-Med. Elect., BME-8, 68 (1961)

[10] G. N. Hounsfield Brit. J. Radiol. 46, 1016 (1973)

[11] J. Ambrose Brit. J. Radiol. 46, 1023 (1973)

[12] A. C. Kak and M. Alaney Principles of Computerized Tomographic Imaging. IEEE, New York, NY, (1988)

[13] L. E. Hall Computer Image Processing and Recognition. Academic Press, New York, NY, (1979)

[14] R. Bender, S. H. Bellman and R. Gordan, J. Theor. Biol. bf-29, 483 (1970)

[15] G. Frieder and G. T. Herman, J. Theor. Biol. 33, 189 (1971)

[16] S. H. Bellman, R. Bender, R. Gordan and J. E. Rowe, J. Theor. Biol. 32, 205 (1971)

[17] M. Goitein, Nucl. Instrum. Methods 101, 509 (1972)

[18] G. T. Herman, Comput. Graphycs Image Proc. 1, 123 (1973)

[19] S. Krishnan, S. S. Prabhu and E. V. Krishnamurthy, Inst. J. Syst. Sci. 4, 661 (1973)

[20] R. Gordon, IEEE Trans. Nucl. Sci. 21, 78 (1974)

[21] R. Gordon and G. T. Herman Int. Rev. Cytol. 38, 111 (1974)

[22] R. L. Kashyap and M. C. Mittal, IEEE Trans. Comput. 24, N 9, 915 (1975)

[23] S. Kaczmarz, Bull. Acad. Pol. Sci. Lett. A, 6-8A, 355 (1937)

[24] K. Tanabe, Numer. Math. 17, 203 (1970)

[25] J. M. Oliveira Jr. and L. P. C. Haak, $4^{0}$ Encontro de Pesquisadores e de Iniciação Científica da Universidade de Sorocaba, 298 (2001) 